\title{
Cutaneous metastases in a patient with no previous diagnosis of cancer: diagnostic challenge*
}

\author{
Pedro de Freitas Silva Torraca ${ }^{1}$ \\ Günter Hans Filho ${ }^{2}$
}

\author{
Bruna Corrêa de Castro ${ }^{2}$ \\ Alexandre Moretti de Lima²
}

DOI: http:/ / dx.doi.org/10.1590/abd1806-4841.20175870

\begin{abstract}
On rare occasions, skin lesions are the first local of metastatic manifestation of internal malignancies. In case of no previous diagnosis of these tumors, the approach of suspicious skin lesions becomes a challenge, especially in differentiating cutaneous metastases and adnexal primary neoplasms. Currently, besides epidemiologic, dermoscopic and histopathologic aspects, the evaluation also integrates immunohistochemical exams and cell markers such as p40 and p63, highly specific for skin metastases. This article describes the case of cutaneous metastases as the sole obvious sign of breast cancer in a previously asymptomatic woman. The diagnosis was made by the finding of neoplastic cells in the dermis and immunohistochemistry compatible with ductal carcinoma.
\end{abstract}

Keywords: Neoplastic metastasis; Neoplastic micrometastasis; Neoplasms, unknown primary

\section{INTRODUCTION}

Cutaneous metastases are uncommon manifestations of internal malignancies and result of the distribution of malignant cells in the skin around and distant from the primary tumor. They can indicate an advanced or recurrent neoplasia, or even represent the sole obvious sign of malignancy. In female patients, breast cancer is the most frequent, followed by lung and colorectal. Identifying these manifestations in patients with no previous diagnosis of a primary neoplasia, as well as the appropriate management of these uncommon lesions, is a real challenge for the dermatologist.

\section{CASE REPORT}

An 83-year-old female patient sought medical care initially because of actinic keratoses. During examination, besides the presenting complaint, three erythematous, well-defined, symmetrical nodules, with approximately $3 \mathrm{~cm}$ in diameter were seen on the left hypochondrium, interscapular region and sacrum (Figures 1 and 2).
She did not know when the lesions first appeared. On physical examination, the patient presented with good general condition and no abnormalities of note.

During the assessment of the lesions, histopathology favored the diagnosis of cutaneous metastases of breast cancer and the immunohistochemistry suggested the ductal subtype (Figures 3 and 4). During follow-up, axillary lymphadenopathy and a subtle fibroelastic and ill-defined nodule in the external upper quadrant of the right breast were noted.

Subsequently, imaging studies were requested and demonstrated metastatic involvement of multiple sites. Computed tomography revealed a $4-\mathrm{mm}$ nodular opacity in the lateral basal segment of the right lung; a 2.5-cm nodular lesion in the right lobe of the thyroid and nodular calcifications on the left lobe; diffuse involvement of the thoracic cage and segments of the spine with a lytic and osteoblastic appearance; enlarged axillary lymphnodes and mediastinal

Work submitted on 16.05 .2016

Approved by the Advisory Board and accepted for publication on 26.09.2016

* Study conducted at Hospital Universitário Maria Aparecida Pedrossian, da Universidade Federal de Mato Grosso do Sul (Humap-UFMS) - Campo Grande (MS), Brazil.

Financial support: None

Conflict of interest: None

Department of Internal Medicine of the Faculdade de Medicina da Universidade Federal de Mato Grosso do Sul (Famed-UFMS) - Campo Grande (MS), Brazil. Dr. Günter Hans Department of Dermatology, at Hospital Universitário da Universidade Federal de Mato Grosso do Sul (NHU-UFMS) - Campo Grande (MS), Brazil.

(C2017 by Anais Brasileiros de Dermatologia 
lymphnodes in the upper limit of normal. The patient was referred to the department of Oncology, where she is undergoing palliative clinical treatment.

\section{DISCUSSION}

Cutaneous metastases are uncommon manifestations related to the direct invasion or local or distant spread of primary malignancies. With an incidence between $0.7 \%$ and $9 \%$ of all cancer cases, they can indicate an advanced or recurrent neoplasia, or even appear as a primary manifestation of malignancy. Breast cancer cutaneous metastasis is the most frequent, however, it is rare to occur as the first sign of this neoplasia.

In 2013, an important review of cutaneous metastasis cases was performed in a Brazilian hospital, with a total of 209 entries in 45 years. This study demonstrated a higher frequency of lesions on the skin of the chest (31.94\% of cases), followed by the abdomen $(20.37 \%)$ and scalp (14.81\%). In women, the most often diagnosed primary malignancy was of the breast (63.19\%), intestines (10.41\%) and lung

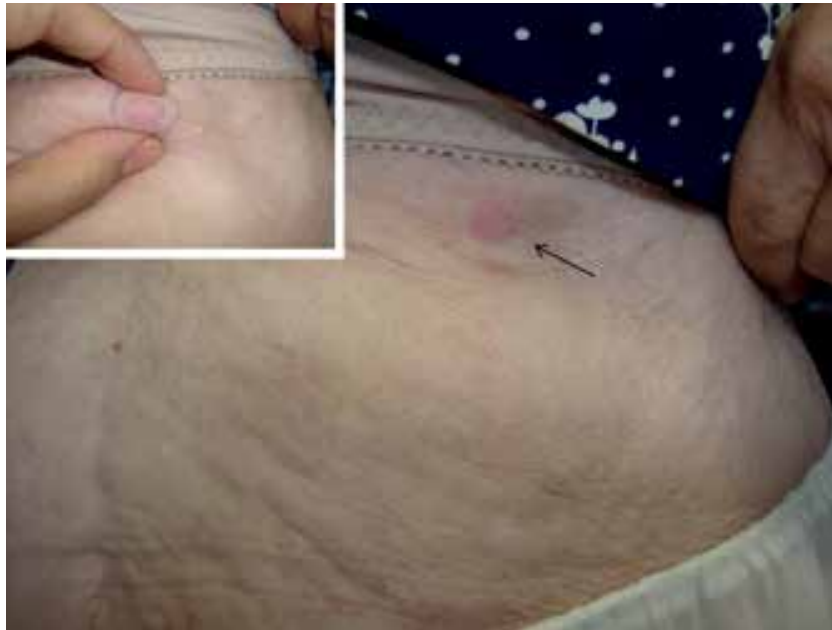

Figure 1: Subcutaneous nodule on the left hypochondrium
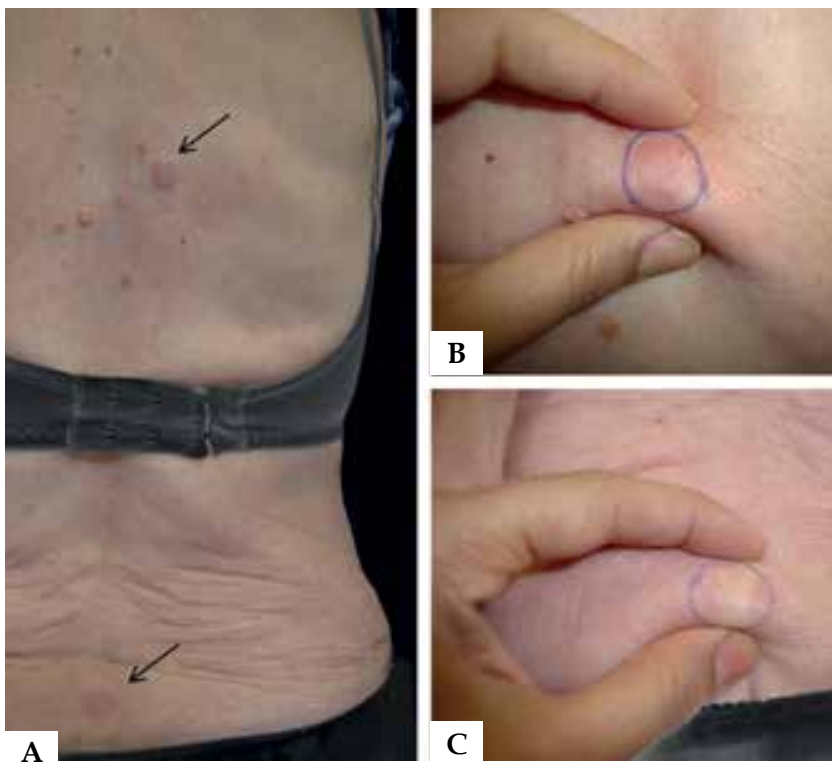

Figure 2: Subcutaneous nodules on the interscapular region and sacrum A. Subcutaneous nodule on the interscapular region $\mathbf{B}$. Subcutaneous nodule on the sacrum $\mathrm{C}$.

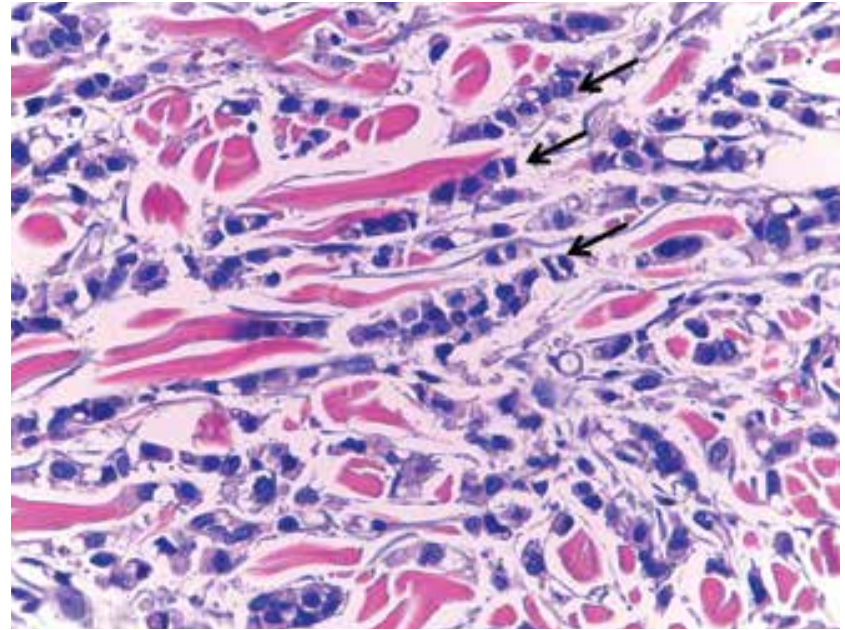

FIGURE 3: Cells with atypical small nuclei, attempting to form lines amid a dense collagenous stroma in the dermis (Hematoxylin \& eosin, X100)
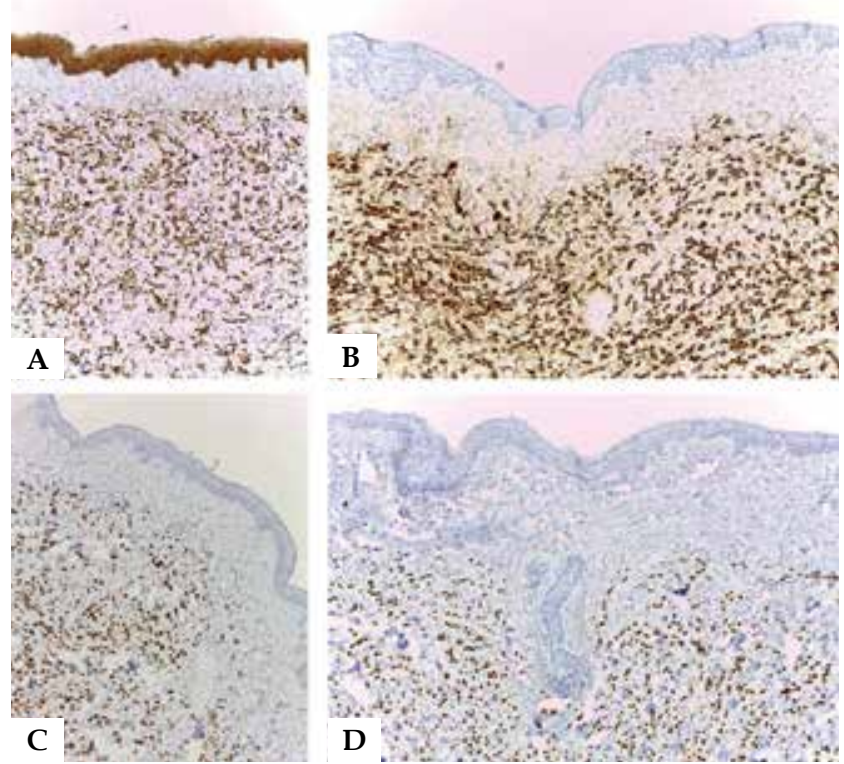

Figure 4: Diffusely positive immunohistochemistry to AE1 + AE2

A; CK7 B; estrogen receptors C; and progesterone receptors

D (immunohistochemistry, X10) 
(4.16\%). In men, lung (33.84\%), stomach (12.30\%) and larynx (7.69\%). ${ }^{2}$

A second study in 2014, conducted for 25 years with 401 cases of cutaneous metastases in the United States demonstrated in absolute numbers the breast as the main site of primary malignancy, corresponding to $32.7 \%$ of the cases of cutaneous metastasis, followed by the bronchi and lungs with $13.2 \%$ of cases. In the breast, the main malignant subtypes seen were the infiltrating ductal carcinoma, in 86 of the 131 cases, and the lobular carcinoma, in 19. Regarding the primary lung malignancies, the adenocarcinoma subtype was responsible for 21 of the 53 cases reported. ${ }^{3}$

For cutaneous metastases, single or multiple nodular lesions are the most common presentations, followed by papules, erysipelas-like, alopecic and sclerotic plaques. They are localized and can present in a zosteriform pattern, especially on the anterior and lateral thorax..$^{2,4,5}$ In a review of 72 cases of cutaneous metastasis, a solitary nodule was seen in $27 \%$ of the total, followed by multiple nodules in $23 \%$. In half of the cases, the lesions were found on the thorax. In women, $81 \%$ of the metastases were from breast carcinoma; in men, $18 \%$ were from neoplasia of the larynx, being them the most common ones. ${ }^{6}$

During the assessment of suspicious nodular lesions, vascular structures, such as microtraumas and violaceous dots in the internal perimeter of the metastasis are frequently seen on dermoscopy. In 20 patients with a histopathological diagnosis of cutaneous metastasis, most lesions were pink or erythematous in color, however, hyperpigmentation was described in three. Of the 17 non-pigmented lesions, $88 \%$ had small arborizing, linear or serpiginous ves- sels, and $12 \%$ had a homogenous pink appearance with no vascular structures. The three hyperpigmented nodular lesions were related to breast carcinoma, corresponding to a total of $60 \%$ of its five cases. ${ }^{7}$

Histopathology remains as the main approach for cutaneous metastatic lesions: it is the gold standard for diagnosis, with adenocarcinomas being the most common tumor type. An analysis of 124 cases of metastasis in Taiwan revealed this characteristic in $76.6 \%$. For the primary malignancies of the breast, ductal and lobular subtypes are the most frequent. In the case of primary malignancy of the lung, the small cell subtype continues to be the most prevalent. ${ }^{8,9}$

The distinction between cutaneous metastasis and primary skin adnexal tumor is, therefore, an important part of the assessment of these lesions. A study with 143 cases of cancer - 76 of those with cutaneous metastasis and 67 with primary skin adnexal tumors - compared the expression of the markers p40, p63, cytokeratin 5/6 (CK5/6) and GATA3. These markers were seen in $80 \%$, $84 \%, 86 \%$ and $47 \%$ of the primary skin adnexal tumors, respectively, and in $8 \%, 17 \%, 26 \%$ e $40 \%$ of metastases. The study demonstrated the statistical significance of the use of p40, p63 and CK5/ 6 in distinguishing these lesions, since these markers had a sensitivity of $80 \%$, $84 \%$ and $86 \%$ for primary skin adnexal tumor and specificity of $92 \%$, $83 \%$ and $74 \%$ for cutaneous metastasis. ${ }^{10}$

The presence of cutaneous metastases in cases of breast malignancy is a sign of poor prognosis, with a mean survival of 3 months. ${ }^{4}$ Therefore, the multidisciplinary evaluation becomes relevant for confirmed metastatic lesions, so as to map the extension of the process and start adequate treatment. ${ }^{3}$

\section{REFERENCES}

1. Prabhu S, Pai SB, Handattu S, Kudur MH, Vasanth V. Cutaneous metastases from carcinoma breast: The common and the rare. Indian J Dermatol Venereol Leprol. 2009:75:499-502.

2. Sittart JA, Senise M. Cutaneous metastasis from internal carcinomas: a review of 45 years. An Bras Dermatol. 2013;88:541-4.

3. Wong CY, Helm MA, Helm TN, Zeitouni N. Patterns of skin metastases: a review of 25 years, experience at a single cancer center. Int J Dermatol. 2014;53:56-60.

4. Virmani NC, Sharma YK, Panicker NK, Dash KN, Patvekar MA, Deo KS. Zosteriform skin metastases: clue to an undiagnosed breast cancer. Indian J Dermatol. 2011;56:726-7.

5. Fernandez-Flores A. Cutaneous metastases: a study of 78 biopsies from 69 patients. Am J Dermatopathol. 2010;32:222-39.

6. El Khoury J, Khalifeh I, Kibbi AG, Abbas 0. Cutaneous metastasis: Clinicopathological study of 72 patients from a tertiary care center in Lebanon. Int J Dermatol. 2014;53:147-58

7. Chernoff KA, Marghoob AA, Lacouture ME, Deng L, Busam KJ, Myskowski PL. Dermoscopic findings in cutaneous metastases. JAMA Dermatol. 2014;150:429-33.
8. Hu SC, Chen GS, Wu CS, Chai CY, Chen WT, Lan CC. Rates of cutaneous metastases from different internal malignancies: experience from a Taiwanese medical center. J Am Acad Dermatol. 2009;60:379-87.

9. Alcaraz I, Cerroni L, Rütten A, Kutzner H, Requena L. Cutaneous metastases from internal malignancies: a clinicopathologic and immunohistochemical review. Am J Dermatopathol. 2012;34:347-93.

10. Lee JJ, Mochel MC, Piris A, Boussahmain C, Mahalingam M, Hoang MP. p40 exhibits better specificity than p63 in distinguishing primary skin adnexal carcinomas from cutaneous metastases. Hum Pathol. 2014;45:1078-83.

\author{
MAILING ADDRESS: \\ Pedro de Freitas Silva Torraca \\ Av. Senador Filinto Müller, 1 \\ Pioneiros \\ 79080-190 Campo Grande, MS \\ Brazil \\ E-mail:pftorraca@gmail.com
}

How to cite this article: Torraca PFS, Castro BC, Hans Filho G, Lima AM. Cutaneous metastases in a patient with no previous diagnosis of cancer: diagnostic challenge. An Bras Dermatol. 2017;92(5 Suppl 1): 47-9. 